\title{
SIOP/COG/NWTSG Stage IV
}

National Cancer Institute

\section{Source}

National Cancer Institute. SIOP/COG/NWTSG Stage IV. NCI Thesaurus. Code C140307.

Presence of hematogenous metastases or metastases to distant lymph nodes. 